\title{
Politisches Wissen in Deutschland. Ein Vergleich von Bürgern mit türkischem Migrationshintergrund und einheimischen Deutschen*
}

\author{
Bettina Westle
}

\section{Normative Bedeutung politischen Wissens für die Demokratie}

Seit der Aufklärung gilt politisches Wissen als Voraussetzung für die Mündigkeit der Staatsbürger. Auch in heutigen repräsentativen Demokratien gehört politische Informiertheit der Bürger zum angestrebten Bürgerschaftsverständnis. Durchgehend wird angenommen, dass Demokratie ohne jegliche Information nicht funktionieren kann und ein guter Informationsstand die Funktionsweise verbessert. Daher zeichnen moderne normative Theorien der Demokratie zumeist ein Bild von bestens informierten, politisch kenntnisreichen Bürgern, die wohlbegründete Präferenzen haben, diese klar ordnen und selbstbewusst vertreten. Dazu gehört, dass sie die politische Agenda kennen und wissen, welche Akteure ihre Interessen aufnehmen oder aber ihnen entgegenstehen. Politische Kenntnisse sind schließlich auch erforderlich, um die Handlungen der politischen Eliten verstehen und kontrollieren zu können und noch offensichtlicher, um selbst effektiv politisch aktiv zu sein. ${ }^{1}$

Allerdings bildet Politik keineswegs für alle Menschen permanent oder auch nur phasenweise den Mittelpunkt ihres Lebens, sondern konkurriert mit vielen anderen Lebensbereichen und Interessen. ${ }^{2}$ Daraus folgt die Vermutung, dass es ebenso wie bei jedem anderen Wissensgebiet erhebliche Unterschiede im politischen Kenntnisstand zwischen den Menschen gibt. Sich über Politik zu informieren oder aber eben nicht, gehört einerseits zur Freiheit in der Demokratie. Andererseits beeinträchtigen individuelle Unterschiede in der politischen Kompetenz jedoch die politische Chancengleichheit. Dies ist insbesondere dann problematisch, wenn unterschiedliche Wissensstände nicht zufällig verteilt sind, sondern an andere strukturelle Merkmale gebunden sind, wenn also ganze Bevölkerungsgruppierungen systematisch und permanent kognitive Defizite im Vorfeld politischer Beteiligung aufweisen.

Insofern die staatsbürgerliche Rolle nur dann effektiv ausgefüllt werden kann, wenn die Bürger über hinreichendes politisches Wissen und Verständnis politischer Vorgänge verfügen, stellt breit verteilte politische Kompetenz schließlich ebenfalls einen zentralen Einflussfaktor für die Qualität der Demokratie insgesamt dar.

* In diesem Beitrag wird der Einfachheit halber nur die männliche Form verwendet. Die weibliche Form ist selbstverständlich immer eingeschlossen.

1 Vgl. Stephen E. Bennett, Trends in American's Political Information, 1967-1987, in: American Politics Quarterly, 17. Jg. (1989), H. 4, S. 422 - 435; Michael X. Delli Carpini / Scott Keeter, What Americans Know About Politics and Why It Matters, Yale 1996; William A. Galston, Political Knowledge, Political Engagement, and Civic Education, in: Annual Review of Political Science, 4. Jg. (2001), S. 217 - 234; Benjamin Barber, Strong Democracy. Participatory Politics for a New Age, Berkeley / Los Angeles / London 2003.

2 Vgl. Philip E. Converse, Popular Representation and the Distribution of Information, in: John H. Ferejohn I James H. Kuklinski (Hrsg.), Information and Democratic Processes, Urbana 1990, S. 369 - 390; Jan W. van Deth, Das Leben, nicht die Politik ist wichtig, in: Oskar Niedermayer I Bettina Westle (Hrsg.), Demokratie und Partizipation, Opladen 2000, S. 115 - 135. 
Angesichts dieser normativen Bedeutung politischen Wissens ist es erstaunlich, wie dürftig der empirische Forschungsstand dazu in Deutschland ist, während beispielsweise in den USA die Erforschung politischen Wissens seit langem zu den Standardthemen der Politikwissenschaft gehört und es sowohl umfassende Querschnitts- als auch Längsschnittstudien dazu gibt sowie eine Vielzahl experimenteller Arbeiten. ${ }^{3}$ In der Bundesrepublik finden sich Arbeiten zu politischen Kenntnissen in der Kommunikationswissenschaft, wobei der Fokus hier auf Medieneinflüssen liegt. ${ }^{4}$ Darüber hinaus gibt es eine Reihe von Studien zu Kindern und Jugendlichen, häufig aus der politischen Bildung und Didaktik, gelegentlich aber auch aus politikwissenschaftlicher Perspektive. ${ }^{5}$ Zur erwachsenen Bevölkerung sind nur im Umfeld von Bundestagswahlen punktuell einige Untersuchungen zu wahlrelevantem Wissen veröffentlicht worden. ${ }^{6}$ Und schließlich finden sich, erneut aus der Perspektive der politi-

3 So urteilt beispielsweise Jeffrey J. Mondak: „Political knowledge has attained the status of a cornerstone construct in research on political behaviour" (ders., Developing Valid Knowledge Scales, in: American Journal of Political Science, 45. Jg. (2001), H. 1, S. $224-238$, S. 238). An dieser Stelle kann der Stand der US-amerikanischen Forschung nicht referiert werden. Vgl. aber dazu zum Beispiel Jürgen Maier, Was die Bürger über Politik (nicht) wissen - und was die Massenmedien damit zu tun haben - ein Forschungsüberblick, in: Barbara Pfetsch / Frank Marcinkowski (Hrsg.), Politik in der Mediendemokratie, Wiesbaden 2009, S. 393 - 414. Eine erste sekundäranalytische Aufarbeitung zu politischem Wissen in Deutschland im Längsschnitt findet sich bei Jürgen Maier / Alexander Glantz / Severin Bathelt, Was wissen die Bürger über Politik? Zur Erforschung der politischen Kenntnisse in der Bundesrepublik Deutschland 1949 bis 2008, in: ZParl, 40. Jg. (2009), H. 3, S. 561 - 579. Auch dort wird resümiert, dass politisches Wissen in Deutschland erst ansatzweise erforscht ist und die vorliegenden Analysen sehr viele Fragen offenlassen müssen sowie neue aufwerfen.

4 Vgl. Reinhold Horstmann, Medieneinflüsse auf politisches Wissen, Wiesbaden 1991; im Überblick: Winfried Schulz, Politische Kommunikation, Theoretische Ansätze und Ergebnisse empirischer Forschung, Opladen 2011.

5 Vgl. international unter Einschluss von Deutschland: Judith Torney-Purta / Rainer Lehman / Hans Oswald / Wolfram Schulz, Citizenship and Education in Twenty-eight Countries: Civic Knowledge and Engagement at Age Fourteen, Amsterdam 2001; Valentin Eck / Georg Weisseno, Political Knowledge of 14- to 15-year-old Students - Results of the TEESAEC Intervention Study in Germany, in: dies. (Hrsg.), Teaching European Citizens. A Quasi-experimental Study in Six Countries, Münster / New York 2009, S. 19 - 30; Thomas Goll / Dagmar Richter / Georg Weisseno / Valentin Eck, Politisches Wissen zur Demokratie von Schüler/innen mit und ohne Migrationshintergrund (POWIS-Studie), in: Georg Weisseno (Hrsg.), Bürgerrolle heute. Migrationshintergrund und politisches Lernen, Bonn 2010, S. 21 - 48; aus politikwissenschaftlicher Perspektive: Klaus Rothe, Schüler und Politik: eine vergleichende Untersuchung bayrischer und hessischer Gymnasialschüler, Opladen 1993; Michaela Ingrisch, Politisches Wissen, politisches Interesse und politische Handlungsbereitschaft bei Jugendlichen aus alten und neuen Bundesländern, Regensburg 1997; Bettina Westle, Politisches Interesse, subjektive politische Kompetenz und politisches Wissen - Eine Fallstudie mit Jugendlichen im Nürnberger Raum, in: Edeltraud Roller / Frank Brettschneider / Jan W. van Deth (Hrsg.), Jugend und Politik: „Voll normal!“ Der Beitrag der politischen Soziologie zur Jugendforschung. Wiesbaden 2006, S. 209 - 240; Jan W. van Deth I Simone Abendschön / Julia Rathke / Meike Vollmar, Kinder und Politik, Wiesbaden 2007.

6 Vgl. Rüdiger Schmitt-Beck, Denn sie wissen nicht, was sie tun... Zum Verständnis des Verfahrens der Bundestagswahl bei westdeutschen und ostdeutschen Wählern, in: ZParl, 24. Jg. (1993), H. 3, S. 393 - 415; Jürgen Maier, Politisches Interesse und politisches Wissen in Ost- und Westdeutschland, in: Jürgen W. Falter / Oscar W. Gabriel / Hans Rattinger (Hrsg.), Wirklich ein Volk? Die politischen Orientierungen von Ost- und Westdeutschen im Vergleich, Opladen 2000, S. 141 - 171; Angelika Vetter / Jürgen Maier, Mittendrin statt nur dabei? Politisches Wissen, politisches Interesse und politisches Kompetenzgefühl in Deutschland, 1994-2002, in: dies. (Hrsg.), 
schen Bildung, angesichts der Selbstanerkennung Deutschlands als Einwanderungsland und des eingeführten Einbürgerungstests einige Arbeiten zum politischen Lernen bei Migranten. ${ }^{7}$

Vor diesem Hintergrund ist ein Forschungsprojekt entwickelt worden, das sich ausschließlich dem politischen Wissen hierzulande sowie seinen Determinanten und Folgen widmet. ${ }^{8}$ Ziel war es, erste Erkenntnisse über den Stand und die Struktur des politischen Wissens der Bevölkerung anhand einer Querschnittsbefragung zu gewinnen. Der Fokus lag dabei auf Faktenwissen, da dieses als notwendige, wenn auch nicht hinreichende Voraussetzung für anspruchsvollere Formen des kognitiven Zugangs zu Politik gelten kann. Zentral waren dabei die Fragen, in welchen Bereichen sich die Deutschen gut auskennen und wo es Wissenslücken gibt, ob politisches Wissen eher generalisiert strukturiert oder eher spezialisiert ist und wie politisches Wissen in verschiedenen Bevölkerungsgruppen ausfällt. Darüber hinaus wird untersucht, welche Faktoren besonders wichtig für den Erwerb beziehungsweise Erhalt politischen Wissens sind, und inwieweit sich politisches Wissen auf politische Einstellungen und politisches Verhalten auswirkt - denn nur wenn letzteres der Fall ist, kommt ihm auch empirisch eine wichtige Bedeutung für die Funktionsfähigkeit der Demokratie zu.

\section{Studienskizze}

Die Erhebung wurde in den Jahren 2008/2009 telefonisch durch Infratest dimap durchgeführt. Sie ist aufgeteilt in einen Studienteil mit einheimischen Deutschen und einen mit Migranten türkischen Hintergrunds. Migranten wurden als Zielgruppe ausgewählt, weil sich durch das neue Einbürgerungsrecht Deutschlands auch die Rolle der Einbürgerungswilligen und Eingebürgerten in der Politik verändert, also größere Chancen für, aber auch höhere Ansprüche an sie als Bürger in der repräsentativen Demokratie bestehen. Dabei fiel die Entscheidung auf Personen mit türkischem Migrationshintergrund, weil diese eine der größten Gruppierungen aus dem Ausland in Deutschland darstellen und sich in der Vergangenheit im Hinblick auf schulische Bildung und Beschäftigung als relativ problematisch erwiesen haben. ${ }^{9}$

Wächst zusammen, was zusammengehört? Stabilität und Wandel politischer Einstellungen im wiedervereinigten Deutschland, Baden-Baden 2005, S. 51 - 90; Bettina Westle (2005), Politisches Wissen und Wahlen, in: Jürgen W. Falter / Oscar W. Gabriel / Bernhard Wessels (Hrsg.), Wahlen und Wähler. Analysen aus Anlass der Bundestagswahl 2002, Wiesbaden 2005, S. 484 - 512; Bettina Westle, Politisches Wissen als Grundlage der Entscheidung bei der Bundestagswahl 2005, in: Steffen Kühnel / Oskar Niedermayer / Bettina Westle (Hrsg.), Wähler in Deutschland. Sozialer und politischer Wandel, Gender und Wahlverhalten, Wiesbaden 2009, S. 366 - 398.

7 Vgl. Oliver Wilhelm / Gizem Hülür / Olaf Köller / Melanie Radalewski, Empirische Grundlagen zum Einbürgerungstest, in: Georg Weisseno (Hrsg.), Bürgerrolle heute. Migrationshintergrund und politisches Lernen, Bonn 2010, S. 49 - 64; ders., Migrationshintergrund und politisches Lernen, in: ebenda, S. 9 - 17.

8 Das Forschungsprojekt wurde von der Thyssen-Stiftung finanziell gefördert. Mitarbeiter waren David Johan und Thomas Schübel.

9 Vgl. Frank Kalter / Nadja Granato / Cornelia Kristen, Disentangling Recent Trends of the Second Generation's Structural Assimilation in Germany, in: Stefanie Scherer / Reinhard Pollack / Gunnar Otte / Markus Gangl (Hrsg.), From Origin to Destination, Frankfurt am Main 2007, S. 214 245. 
Bei dem ersten Studienteil handelt es sich um eine Repräsentativerhebung unter der bundesdeutschen Bevölkerung ab 14 Jahren ohne türkischen Migrationshintergrund in allen westlichen und östlichen Bundesländern sowie in Berlin mit insgesamt 1.630 geführten Interviews. Die Ausschöpfungsquote betrug knapp 49 Prozent. Von den 1.630 Interviews fanden 75 Prozent in West- und 25 Prozent in Ostdeutschland statt. Die nachfolgend präsentierten Analysen sind gewichtet, um die Überrepräsentation Ostdeutschlands auszugleichen. Die Befragungen dauerten im Mittel 28 Minuten, bei einer Spanne zwischen 21 und 62 Minuten.

In der Migrantenstudie wurden 300 Interviews mit Einwohnern türkischen Migrationshintergrunds ab 14 Jahren durchgeführt. Diese Befragung basiert auf einer regional geschichteten Zufallsstichprobe auf Grundlage aktueller Telekomdaten. Eingesetzt wurde das so genannte onomastische Verfahren (Stichprobe anhand von Namensbestandteilen, die für den jeweiligen Sprachraum charakteristisch sind). Die Ausschöpfungsquote lag bei rund 35 Prozent. Von den 300 Befragten verfügen etwa 64 Prozent nur über die türkische, 29 Prozent nur über die deutsche und sechs Prozent über beide Staatsangehörigkeiten. Als primären Lebensraum während der Jugend gaben aber nur 33 Prozent die Türkei, 59 Prozent West- und sechs Prozent Ostdeutschland an. Die Interviewdauer betrug im Mittel 31 Minuten, bei einer Spannweite von 22 bis 62 Minuten. Sichergestellt war sowohl eine türkische als auch eine deutsche Ansprache beim Gesprächseinstieg sowie nachfolgend die Möglichkeit zur Auswahl der bevorzugten Sprache durch die Befragungsperson. Türkisch präferierten 59 Prozent, Deutsch 25 Prozent, und weitere 16 Prozent der Interviews wurden zweisprachig durchgeführt.

\section{Zur Definition politischen Wissens}

Zur Bestimmung des Untersuchungsgegenstandes hat sich das Projekt an Delli Carpini und Keeter - den Autoren der bisher umfangreichsten Arbeit zu diesem Thema in den USA orientiert. Sie definieren politisches Wissen als ,the range of factual information about politics that is stored in long-term memory "10. Durch den Bezug auf Politics wird das Konzept von anderen Wissensinhalten (zum Beispiel Mathematik, Kunst) abgegrenzt. Mit „factual information“ sind faktisch zutreffende Kenntnisse gemeint, auch objektives, deklaratives oder explizites Wissen genannt. ${ }^{11}$ Die Definition grenzt Faktenwissen von solchen Kognitionen ab, die objektiv falsch sind, und von solchen, die nicht valide und reliabel getestet werden können sowie von prozessualen Fähigkeiten des Kenntniserwerbs. Als weiteres Merkmal soll es sich um Informationen handeln, die im Langzeitgedächtnis gespeichert sind, also nicht um neue ad hoc Informationen. Zweifellos ist Faktenwissen allein keine hinreichende Bedingung für ein tieferes Verständnis politischer Vorgänge. Dafür müssen die Informationen zusätzlich in Kontexte eingeordnet werden, Relevanz erlangen und vieles mehr. Wenn jedoch schon die notwendige Bedingung des Faktenwissens nicht erfüllt ist, kann es auch nicht zu einem tieferen Verständnis kommen.

10 Michael X. Delli Carpini / Scott Keeter, a.a.O. (Fn. 1), S. 10.

11 Vgl. Eric R. Kandel / James H. Schwartz / Thomas M. Jessel, Essentials of Neural Science and Behavior, Stanford 1995; Markus Prior / Arthur Lupia, Money, Time, and Political Knowledge: Distinguishing Quick Recall and Political Learning Skills, in: American Journal of Political Science, 52. Jg. (2008), H. 1, S. $169-183$. 
Die dargestellte Definition lässt offen, was Bürger eigentlich wissen sollten. Häufig, auch in der genannten Studie, finden sich drei grundlegende Kategorien von Wissensbereichen, über die die Bürger nach Auffassung der Wissenschaftler informiert sein sollten, damit eine Demokratie funktioniert. Erstens sollten Bürger über „Institutionelles Basiswissen“ verfügen, das heißt wissen, was der Staat ist, was er tut und was die wichtigsten Regeln und Verfahrensweisen in der Politik sind. Um ihre staatsbürgerliche Rolle effektiv ausüben zu können, sollten sie zweitens Wissen über „aktuelle politische und sozioökonomische Themen, Probleme und Streitfragen" haben. Und um sich in alltäglichen Politikdebatten zurecht zu finden und bei Wahlen eine begründete Entscheidung treffen zu können, stellt drittens die Kenntnis von „Parteien und politischen Akteuren“, auch von ihren ideologischen Orientierungen, Standpunkten und Zielen in umstrittenen Fragen sowie ihren Verfahrensvorschlägen bei parteiübergreifend konsensualen Zielen ein weiteres zentrales Element politischen Wissens dar. Darüber hinaus ist eine Vielzahl weiterer Wissensbereiche denkbar, zum Beispiel zur Geschichte oder zu speziellen Interessen.

\section{Probleme der Theorie, der Operationalisierung und der Messung}

Wichtige Kernfragen für die Theoriebildung betreffen die Entwicklung und die Struktur von politischem Wissen. Im Hinblick auf die Entwicklung dürften auf der individuellen Ebene ähnliche Faktoren wie generell beim Wissenserwerb eine Rolle spielen, so vor allem (a) Fähigkeiten (wie Auffassungsvermögen, Intelligenz, Merkfähigkeit), (b) Interesse und Motivation, und zwar im Wechselspiel mit (c) dem Informationsangebot und Gelegenheiten zur Informationsaufnahme. Letzteres ist auch für die kollektive Ebene zentral. Empirisch zeigen Delli Carpini und Keeter für die Bevölkerung der USA und jüngst auch Maier, Glantz und Bathelt auf der Grundlage von Sekundäranalysen für Deutschland, dass trotz des Anstiegs der allgemeinen Bildungsstände und der Verbreiterung des medialen Informationsangebots der politische Kenntnisstand unter der Bevölkerung seit Jahrzehnten weitgehend stabil geblieben ist. ${ }^{12}$

Viele Menschen verlassen sich auf so genannte Heuristiken, um zu politischen Entscheidungen zu kommen. Ob auf solche Weise gewonnene Annahmen allerdings immer richtig sind und zu vernünftigen Entscheidungen führen, darf bezweifelt werden. Es wurde nämlich beobachtet, (a) dass bei Fehlinformationen oder einem Mangel an politischen Kenntnissen andere politische Urteile und Entscheidungen getroffen werden als bei einem guten Wissensstand, (b) dass politisches Faktenwissen dabei hilft, neue Informationen aufzunehmen und falsche Informationen zu verwerfen, und (c) dass Menschen mit einem guten Kenntnisstand auch von der Anwendung von Heuristiken mehr profitieren als solche mit einem schlechten Kenntnisstand. ${ }^{13}$ Für die Theorie folgt aus diesen Beobachtungen die

12 Vgl. Michael X. Delli Carpini / Scott Keeter, a.a.O. (Fn. 1); Jürgen Maier / Alexander Glantz / Severin Bathelt, a.a.O. (Fn. 3).

13 Vgl. beispielsweise Martin Gilens, Political Ignorance and Collective Policy Preferences, in: American Political Science Review, 95. Jg. (2001), H. 2, S. 379 - 396; Vincent Price / John Zaller, Who Gets the News? Alternative Measures of News Reception and Their Implications for Research, in: Public Opinion Quarterly, 57. Jg. (1993), H. 2, S. 133 - 164; Samuel Popkin I Michael A. Dimock, Political Knowledge and Citizen Competence, in: Stephen L. Elkin / Karol E. Soltan (Hrsg.), Citizen Competence and Democratic Institutions, University Park 1999, S. 117 
Frage, ob und wie sich das politische Wissen der Bevölkerung steigern lässt, um die Qualität der Demokratie zu verbessern, oder ob die Demokratie umgekehrt so gestaltet werden müsste, dass sie mit eher mäßig kompetenten Bürgern funktioniert und dabei noch ihren Namen verdient.

Zur Struktur politischen Wissens stellt sich auf der individuellen Ebene die Frage, ob das Wissen spezialisiert oder generalisiert vorliegt. Eine spezialisierte Wissensstruktur bedeutet, dass Individuen dazu tendieren, sich nur in einem oder wenigen bestimmten Bereichen besonders gut auszukennen, etwa wenn diese besondere Bedeutung für ihre eigenen Lebensumstände haben. Eine generalistische Wissensstruktur liegt vor, wenn Personen dazu neigen, sich auf allen politischen Gebieten entweder gut oder schlecht auszukennen. Auf kollektiver Ebene stellt sich die Frage nach Mustern und Verteilungen des Wissens in verschiedenen Bevölkerungsgruppen, beispielsweise aufgegliedert nach Schichten, Alter oder Geschlecht. Beide Ebenen sind eng miteinander verknüpft, insbesondere wenn spezialisierte Wissensstrukturen vorliegen, die durch unterschiedliche Lebensumstände geprägt werden.

Die Frage nach der Struktur hat unmittelbare Auswirkungen auf die Möglichkeiten der Operationalisierung und Messung von politischem Wissen in allgemeinen Bevölkerungsbefragungen, denn im Fall spezialisierter Wissensstrukturen genügt ein einfacher Index von Wissensfragen, der nur wenige politische Felder abdeckt, nicht, um generelle Informationen über den politischen Gesamtwissensstand einer Person zu erhalten. Sollte das Wissen dagegen generalistisch sein, könnten durch eine kluge Auswahl nur weniger Fragen gute Aussagen über generelle Wissensstände auch in anderen Bereichen getroffen werden. Da hierüber noch keine empirischen Informationen vorlagen, wurde in dem Projekt versucht, eine möglichst große Breite von Wissensbereichen anzusprechen, die potenziell spezialisierte Strukturen erfassen können sollten (zum Beispiel Fragen, von denen man annehmen konnte, dass sie für Frauen oder aber Männer, für Jüngere oder aber Ältere, für einheimische Deutsche oder aber für Migranten besondere Bedeutung haben).

Eine weitere methodische Kontroverse dreht sich um offene oder geschlossene Fragen. Erstere lassen den Befragten mehr Spielraum in der Beantwortung, sind jedoch auch anspruchsvoller (Wissensaktivierung statt nur Reaktion) und zeitaufwendiger. Zudem hat auch der Interviewer unerwünschten Spielraum beim Notieren der Antworten. MultipleChoice-Fragen bergen die Gefahr, dass durch die Antwortvorgaben ungewollt Hinweise auf die richtige Antwort gesetzt werden. Auch über die günstigste Anzahl an Antwortvorgaben schwanken die Empfehlungen zwischen drei und fünf. Eine hohe Anzahl erhöht den Schwierigkeitsgrad der Frage und reduziert Zufallstreffer. Eine geringe ist aber in telefonischen Umfragen besser zu implementieren, denn die Befragten vergessen dann die Antwortmöglichkeiten nicht so leicht. Ferner ist es bei manchen Themen kaum möglich, eine höhere Anzahl plausibler Antwortvorgaben zu formulieren. ${ }^{14}$

Strittig ist auch, ob die Bekundung fehlenden Wissens ermutigt werden soll, um die Rateneigung zu reduzieren, oder ob die Befragten zu inhaltlichen Antworten gedrängt werden sollen, um kognitionsfremde Einflüsse wie Selbstbewusstsein, Risikofreude und Wett-

- 146; Richard R. Lau / David P. Redlawsk, Advantages and Disadvantages of Cognitive Heuristics in Political Decision Making, in: American Journal of Political Science, 45. Jg. (2001), H. 4, S. $951-971$.

14 Vgl. Jeffrey J. Mondak, a.a.O. (Fn. 3). 
bewerbsorientierung auszuschalten. Während erziehungswissenschaftliche Studien zumeist versuchen, die Rateneigung der Respondenten auf ein identisches Niveau zu bringen, ermutigen politikwissenschaftliche Untersuchungen in der Regel die Option „weiß nicht“. Ziel dabei ist es, nicht nur das Raten zu reduzieren, sondern zu vermeiden, dass schlecht informierte Respondenten demotiviert werden. Außerdem kann die Option „weiß nicht“ auch inhaltlich anders interpretiert werden als fehlerhafte Kenntnisse, denn bei letzteren ist der Befragte von seinem Wissen überzeugt, unterliegt dabei aber einem Irrtum, während ihm bei ersterem sein Wissensmangel bewusst ist - dieser Unterschied dürfte vor allem im Hinblick auf politisches Handeln verschiedene Konsequenzen zeitigen. ${ }^{15}$ Vor diesem Hintergrund wurden für die vorliegende Studie unterschiedliche Instrumente entwickelt: eine offene Frage zur symbolischen Politik (Kenntnis des Anfangs der Nationalhymne), ein Multiple-Choice-Quiz zu institutionellem Wissen, zu Politikfeldern und gesellschaftlichen Fakten (mit drei Antwortvorgaben, da sich vier im Pretest als ungünstig herausgestellt hatten), eine offene Fragebatterie zur Zuordnung von Parteien zu Politikern und vier Fragen zu Parteipositionen in umstrittenen Themen (siehe Abbildung 1). ${ }^{16}$

\section{Abbildung 1: Übersicht der gestellten Fragen}

Neben der Fahne ist die Nationalhymne eines der wichtigsten Symbole eines Landes. Können Sie spontan den Anfang der deutschen Nationalhymne wiedergeben?

vollständige und korrekte Antwort („Einigkeit und Recht und Freiheit für das deutsche Vaterland“); unvollständige, aber zum Teil korrekte Antwort (zum Beispiel „Einigkeit und Recht und Freiheit ..."); alte Version des Deutschlandlieds in jeder Form (zum Beispiel „Deutschland, Deutschland über alles, über alles in der Welt"); Sonstiges; weiß nicht; keine Angabe

\section{Wen vertreten Gewerkschaften in Deutschland?}

Unternehmer; Arbeitnehmer; Selbständige

Wie viele verschiedene Pflegestufen sieht das System der Pflegeversicherung vor?

drei; vier; fünf

Bei welchen Wahlen dürfen EU-Bürger, die in Deutschland leben, wählen, auch wenn sie nicht die deutsche Staatsbürgerschaft haben?

bei Kommunalwahlen; bei Bundestagswahlen; bei Landtagswahlen

\section{Was versteht man unter „dynamischer Rente“?}

die Höhe der Rente ist an das aktuelle Lohnniveau gekoppelt; die Rentner haben die Wahl zwischen staatlicher und privater Altersvorsorge; das Renteneintrittsalter ist flexibel

Wie hoch ist die Ausgabensumme des Bundeshaushalts zurzeit ungefähr? 20 bis 25 Milliarden; 250 bis 350 Milliarden; 800 bis 900 Milliarden

Ab welchem Alter darf man in Deutschland als Kandidat für den Bundestag antreten? 16 Jahre; 18 Jahre; 21 Jahre

15 Vgl. Malcolm Slakter, Generality of Risk Taking on Objective Examinations, in: Educational and Psychological Measurement, 29. Jg. (1969), S. 115 - 128; Richard Nadeau / Richard G. Niemi, Educated Guesses: The Process of Answering Factual Knowledge Questions in Surveys, in: Public Opinion Quarterly, 59. Jg. (1995), H. 3, S. 323 - 346; Jeffrey J. Mondak / Belinda Creel Davis, Asked and Answered: Knowledge Levels When We Will Not Take "Don't Know" For an Answer, in: Political Behavior, 23. Jg. (2001), H. 3, S. $199-224$.

16 Näheres zu den Wissensfragen kann bei der Autorin angefordert werden. Ausgewertet werden an dieser Stelle nur die Anteile richtiger Antworten. 


\section{Fortsetzung Abbildung 1}

Wie hoch ist derzeit in etwa die Arbeitslosenquote in Deutschland?

5 Prozent; 7 Prozent; 12 Prozent

Wie hoch ist der Ausländeranteil in Deutschland derzeit in etwa?

4 Prozent; 9 Prozent; 15 Prozent

Wie viele Länder sind zurzeit Mitglied der Europäischen Union?

$18 ; 27 ; 32$

\section{Welche Aufgabe hat der Bundesrat?}

der Bundesrat wählt den Bundespräsidenten; durch den Bundesrat wirken die Länder bei der Gesetzge-

bung und Verwaltung des Bundes mit; der Bundesrat wählt den Bundeskanzler

Das Grundgesetz bestimmt die Grundlagen des politischen Systems in Deutschland.

Alle Staatsgewalt geht demnach aus ...?

vom Bundesverfassungsgericht; vom Volk; von der Bundesregierung

Wie hoch ist derzeit in etwa der Anteil der Frauen im erwerbsfähigen Alter, die auch tatsächlich erwerbstätig sind?

45 Prozent; 65 Prozent; 75 Prozent

\section{Gegen was richtet sich die europäische Anti-Diskriminierungsrichtlinie?}

Benachteiligungen von Arbeitnehmern im Berufsleben; Gewalt gegenüber Migranten; Benachteiligung von ausländischen Firmen

Wieviel Prozent des Bruttoinlandsprodukts hat Deutschland im letzten Jahr für Entwicklungshilfe verwendet?

0,4 Prozent; 2,8 Prozent; 7,2 Prozent

Was wollte die Regierung Adenauer mit der Hallstein-Doktrin erreichen?

Nicht-Anerkennung der DDR durch andere Staaten; Beitritt des Saarlandes zur Bundesrepublik; Wiederbewaffnung der Bundeswehr

Was versteht man unter dem „Bologna-Prozess“?

Einführung von vergleichbaren Studienabschlüssen in vielen europäischen Ländern; Schaffung einer gemeinsamen europäischen Flüchtlingspolitik; EU-Programm zur Korruptionsbekämpfung

\section{Was regeln die Kopenhagener Kriterien?}

Voraussetzungen für EU-Beitritt; Finanzausgleich zwischen Mitgliedsstaaten der EU; Fangquoten des Europäischen Fischereifonds

Ich nenne Ihnen jetzt einige Namen von Politikern. Bitte sagen Sie mir für jede Person, welcher Partei sie angehört: der CDU/CSU, der SPD, der FDP, den Grünen oder der Linken.

Michael Glos; Ursula von der Leyen; Cem Özdemir; Petra Pau; Olaf Scholz; Guido Westerwelle

Bei der folgenden Frage geht es jeweils um gegensätzliche Meinungen zu verschiedenen politischen Sachfragen. Bitte sagen Sie mir jeweils, welche Meinung Sie persönlich dazu haben und welche Meinung die einzelnen Parteien dazu vertreten. Wenn Sie es nicht wissen, sagen Sie es einfach.

- dass das kommunale Wahlrecht auf Nicht-EU-Ausländer ausgedehnt wird?

- dass die Laufzeit der deutschen Atomkraftwerke verlängert wird?

- dass sozialstaatliche Leistungen abgebaut und die Eigenbeteiligung der Bürger gestärkt wird?

- dass die Türkei die Vollmitgliedschaft in der Europäischen Union erhält?

Parteien jeweils: CDU/CSU; SPD; FDP; Bündnis 90/Die Grünen; Die Linke

Antwortvorgaben jeweils: dafür, dagegen

Anmerkung: Die Reihenfolge der Fragen entspricht ihrem Auftreten in diesem Beitrag und variiert zu der im Fragebogen. Bei den Quizfragen wurden die Antwortvorgaben rotiert (Ausnahme Zahlenvorgaben), ebenso wurde die Personenreihenfolge bei der Frage nach den Politikern rotiert.

Quelle: Eigene Zusammenstellung. 


\section{Ausgewählte Befunde zur Verteilung politischen Wissens}

Um die Verteilung politischen Wissens in unterschiedlichen Gruppierungen der Bevölkerung zu ermitteln, wird exemplarisch der Vergleich von einheimischen Deutschen mit in Deutschland lebenden Personen mit türkischem Migrationshintergrund herangezogen. Beide Gruppen werden nochmals differenziert, und zwar in Einwanderer/-nachkommen mit und solche ohne deutsche Staatsbürgerschaft sowie in West- und Ostdeutsche. Hintergrund der ersten Unterscheidung ist die Annahme, dass die Kenntnisse bei Einwanderern im Schnitt geringer als bei Einheimischen sein dürften, da die Aneignung von politischem Wissen Zeit, Motivation und Sprachkenntnisse voraussetzt, also Faktoren, die bei ersteren sozialisations- und situationsbedingt häufig in geringerem Ausmaß vorhanden sein dürften. Zweitens wird erwartet, dass bei den Ostdeutschen die Kenntnisse insbesondere zur Struktur des für sie relativ neuen politischen Systems im Durchschnitt geringer sind als bei Westdeutschen. Und drittens wird angenommen, dass Migranten, die bereits die deutsche Staatsbürgerschaft erworben haben, stärker motiviert waren, sich mit der deutschen Politik auseinanderzusetzen und dass sie folglich mehr wissen als ihre Mitbürger, die ausschließlich über die türkische Staatsbürgerschaft verfügen. ${ }^{17}$

Bei der singulären offenen Frage zur symbolischen Politik (siehe Tabelle 1) wird deutlich, dass die zuvor angeführten Erwartungen zutreffen: Westdeutsche kennen den Anfangstext der deutschen Nationalhymne häufiger als Ostdeutsche (die bei dieser Frage allerdings auch auffällig häufig die Antwort verweigern), und Migranten haben hier einen schlechteren Kenntnisstand als Einheimische, wobei diejenigen mit deutscher Staatsbürgerschaft besser abschneiden als diejenigen ohne.

Bei den Quizfragen mit drei Antwortvorgaben (siehe Tabelle 2) ist zu berücksichtigen, dass eine etwa 33-prozentige Chance besteht, durch Raten die richtige Antwort zu geben. Vor diesem Hintergrund zeigt sich, dass bei den einheimischen Deutschen vor allem Fragen, die auf die Europäische Union oder das Ausland bezogen sind, besonders schlecht ausfallen. Bei Themen rund um den Arbeitsalltag und Soziales ist der Kenntnisstand dagegen relativ gut. Die Unterschiede zwischen West und Ost sind dabei überwiegend nur gering. Bei der Migrantenpopulation besteht ein vergleichbar großes Wissen nur zu den Gewerkschaften, ob nun als Folge ihres eigenen Arbeitsalltages in Deutschland oder aber, weil Gewerkschaften aus der Türkei bekannt sind. Darüber hinaus ist auffällig, dass bei allen auf Deutschland bezogenen Fragen die Migranten deutlich schlechter abschneiden als die Einheimischen, jedoch nur unwesentlich schlechter bei den auf die EU und das Ausland bezogenen Fragen. In Bezug auf die Aufnahmekriterien in die EU ist das Wissen unter den Migranten sogar verbreiteter als unter den einheimischen Deutschen. Auch besteht bei diesen Fragen kein durchgehender Vorsprung der Migranten mit deutscher Staatsangehörigkeit. Vielmehr scheinen diejenigen mit ausschließlich türkischer Staatsangehörigkeit etwas besser in EUAngelegenheiten informiert. Es ist zu vermuten, dass sich hier ein besonderes Interesse an der Thematik infolge der Debatten um eine Aufnahme der Türkei in die EU widerspiegelt.

17 Optimal sollte hier zwischen den Generationen der Eingewanderten (beziehungsweise neu Eingewanderten und ihren hier aufgewachsenen Nachkommen) oder nach der Dauer des Aufenthalts in Deutschland differenziert werden, da hiervon vor allem die Chancen zum Wissenserwerb abhängen; allerdings ist dies infolge der geringen Befragtenzahl in der Migrantenstudie nicht möglich. 


\begin{tabular}{|l|ccc|ccc|}
\hline Tabelle 1: Kenntnis des Anfangs der deutschen Nationalhymne \\
\hline \multirow{2}{*}{$\begin{array}{l}\text { Prozentanteile richtige } \\
\text { Antworten }\end{array}$} & \multicolumn{2}{|c|}{ deutscher Hintergrund } & \multicolumn{3}{c|}{ türkischer Migrationshintergrund } \\
\cline { 2 - 8 } & gesamt & West & Ost & gesamt & $\begin{array}{c}\text { deutsche Staats- } \\
\text { angehörigkeit }\end{array}$ & $\begin{array}{c}\text { keine deutsche } \\
\text { Staatsangehörigkeit }\end{array}$ \\
\hline Einigkeit und Recht... & 60 & 64 & 41 & 24 & 31 & 21 \\
\hline Quelle: Eigene Berechnung.
\end{tabular}

\section{Tabelle 2: Kenntnis von politischen Strukturen, Politikfeldern und gesellschaftlichen Fakten}

\begin{tabular}{|c|c|c|c|c|c|c|}
\hline \multirow[b]{2}{*}{$\begin{array}{l}\text { Prozentanteile richtige } \\
\text { Antworten }\end{array}$} & \multicolumn{3}{|c|}{ deutscher Hintergrund } & \multicolumn{3}{|c|}{ türkischer Migrationshintergrund } \\
\hline & gesamt & West & Ost & gesamt & $\begin{array}{c}\text { deutsche Staats- } \\
\text { angehörigkeit }\end{array}$ & $\begin{array}{c}\text { keine deutsche } \\
\text { Staatsangehörig- } \\
\text { keit }\end{array}$ \\
\hline $\begin{array}{l}\text { Funktion von } \\
\text { Gewerkschaften }\end{array}$ & 91 & 90 & 92 & 78 & 84 & 75 \\
\hline Bedeutung Pflegestufen & 73 & 74 & 72 & 33 & 44 & 28 \\
\hline EU-Bürger Wahlrecht & 64 & 65 & 60 & 40 & 42 & 40 \\
\hline Dynamische Rente & 63 & 63 & 60 & 27 & 29 & 25 \\
\hline Anzahl EU-Länder & 60 & 60 & 57 & 54 & 48 & 57 \\
\hline Bundeshaushalt & 60 & 62 & 52 & 34 & 40 & 32 \\
\hline Alter Kandidat Bundestag. & 54 & 53 & 58 & 34 & 39 & 33 \\
\hline Arbeitslosenquote & 54 & 56 & 44 & 33 & 35 & 32 \\
\hline $\begin{array}{l}\text { Ausländeranteil in Deutsch- } \\
\text { land }\end{array}$ & 50 & 50 & 50 & 33 & 46 & 27 \\
\hline Aufgabe Bundesrat & 49 & 51 & 39 & 38 & 39 & 38 \\
\hline Staatsgewalt geht aus von & 48 & 48 & 47 & 42 & 41 & 41 \\
\hline Erwerbsquote Frauen & 40 & 39 & 43 & 26 & 33 & 24 \\
\hline $\begin{array}{l}\text { Anti-Diskriminierungsricht- } \\
\text { linie }\end{array}$ & 33 & 35 & 28 & 26 & 19 & 28 \\
\hline Umfang Entwicklungshilfe & 26 & 28 & 20 & 21 & 23 & 21 \\
\hline Hallstein-Doktrin & 23 & 23 & 27 & 16 & 20 & 14 \\
\hline Bologna-Prozess & 23 & 24 & 21 & 15 & 10 & 15 \\
\hline Kopenhagener Kriterien & 22 & 21 & 24 & 49 & 46 & 50 \\
\hline Mittelwert $0-17$ & 8.31 & 8.41 & 7.90 & 5.99 & 6.38 & 5.79 \\
\hline
\end{tabular}

Dass ein heimatlicher beziehungsweise ethnischer Bezug für das politische Kenntnisniveau eine wichtige Rolle spielt, wird noch deutlicher bei der ungestützten Angabe der Parteizugehörigkeiten von Politikern (siehe Tabelle 3). So ist von den bundesdeutschen Politikern, deren Parteizugehörigkeit angegeben werden sollte, dies unter den Bürgern mit türkischem Migrationshintergrund nur für Cem Özdemir mit weitem Abstand vor allen anderen vielen Befragten gut gelungen - ausnahmsweise konnten dies sogar mehr als unter den Einheimischen. Bei den einheimischen Deutschen ist das Kenntnisniveau der Parteizugehörigkeiten dagegen vergleichsweise gut - die Rolle unterschiedlicher Betroffenheits- beziehungsweise Zugehörigkeitsgefühle wird aber auch hier beispielsweise daran deutlich, dass im Osten der Grüne Özdemir deutlich weniger bekannt ist, jedoch Petra Paus Zugehörigkeit zur Linken etwas häufiger als im Westen genannt wurde.

Ein ähnliches Muster findet sich auch in der Verortung der Parteien bei vier Positionsissues (siehe Tabelle 4). Die größere Bekanntheit und Beliebtheit der Linken im Osten 


\begin{tabular}{|l|ccc|ccc|}
\hline Tabelle 3: Kenntnis von Parteizugehörigkeiten prominenter Politiker \\
\hline \multirow{2}{*}{$\begin{array}{l}\text { Prozentanteile richtige } \\
\text { Antworten }\end{array}$} & deutscher Hintergrund & \multicolumn{3}{c|}{ türkischer Migrationshintergrund } \\
\cline { 2 - 7 } & gesamt & West & Ost & gesamt & $\begin{array}{c}\text { deutsche } \\
\text { Staats- } \\
\text { angehörigkeit }\end{array}$ & $\begin{array}{c}\text { keine deutsche } \\
\text { Staats- } \\
\text { angehörigkeit }\end{array}$ \\
\hline Guido Westerwelle (FDP) & 82 & 83 & 81 & 14 & 22 & 10 \\
Cem Özdemir (Grüne) & 65 & 69 & 47 & 66 & 77 & 61 \\
Ursula von der Leyen (CDU) & 63 & 64 & 59 & 19 & 28 & 15 \\
Michael Glos (CSU) & 53 & 54 & 49 & 15 & 24 & 11 \\
Olaf Scholz (SPD) & 43 & 46 & 34 & 8 & 16 & 5 \\
Petra Pau (Die Linke) & 32 & 30 & 38 & 4 & 10 & 2 \\
\hline Mittelwert 0 -6 & 3.37 & 3.45 & 3.08 & 1.26 & 1.76 & 1.04 \\
\hline Quelle: Eigene Berechnung.
\end{tabular}

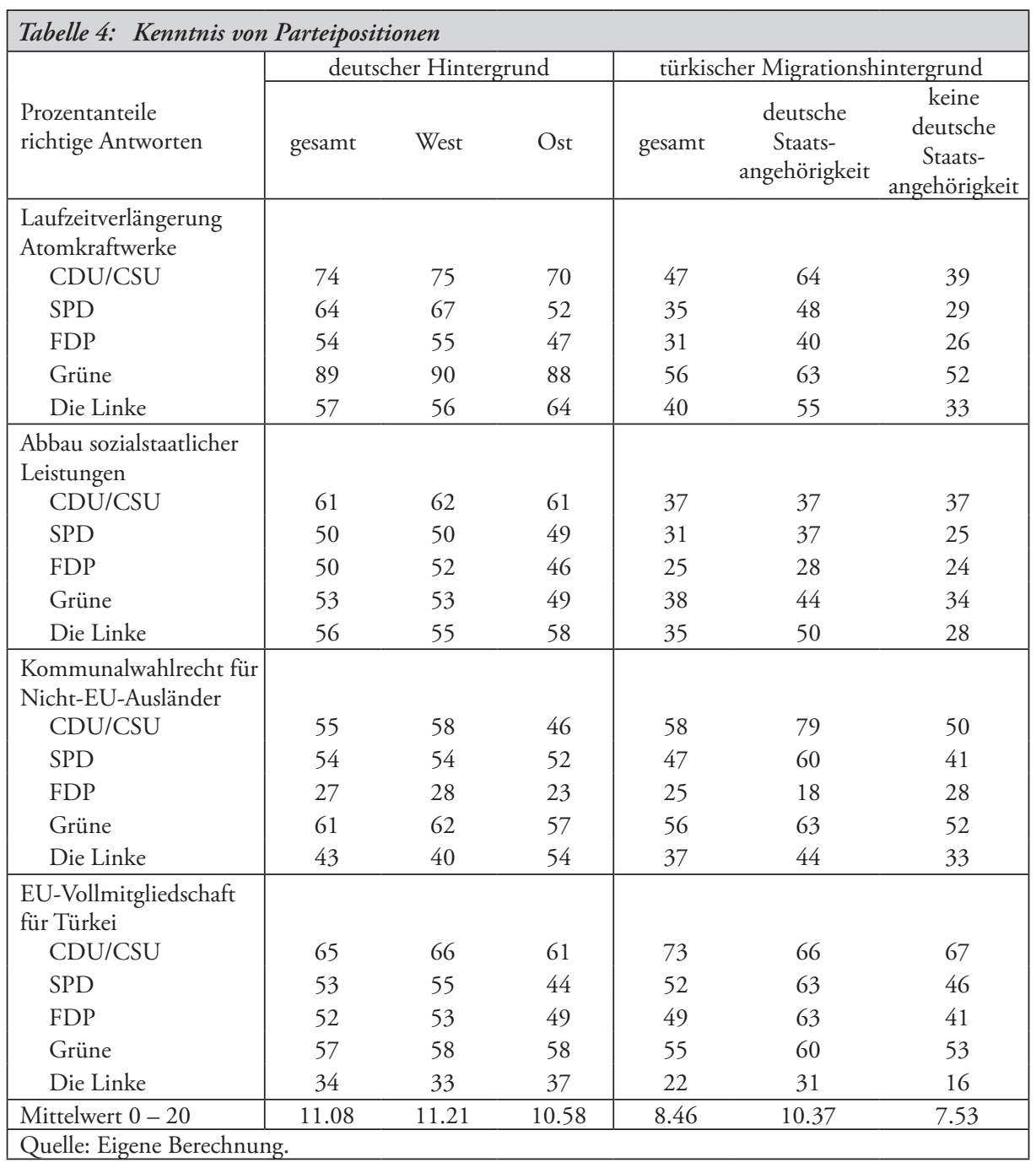




\begin{tabular}{|c|c|c|c|c|c|c|}
\hline \multirow[b]{2}{*}{ Pearsons $\mathrm{r}$} & \multicolumn{3}{|c|}{ deutscher Hintergrund } & \multicolumn{3}{|c|}{ türkischer Migrationshintergrund } \\
\hline & gesamt & West & Ost & gesamt & $\begin{array}{c}\text { deutsche } \\
\text { Staats- } \\
\text { angehörigkeit }\end{array}$ & $\begin{array}{l}\text { keine deutsche } \\
\text { Staats- } \\
\text { angehörigkeit }\end{array}$ \\
\hline Hymne - Quiz & $.18^{* *}$ & $.19^{* *}$ & .12 & $.21^{* *}$ & $.43^{* *}$ & .11 \\
\hline Hymne - Politiker & $.16^{* *}$ & $.16^{* *}$ & $.13^{*}$ & $.25^{* *}$ & $.30^{* *}$ & $.22^{* *}$ \\
\hline Hymne - Issues & $.24^{* *}$ & $.23^{* *}$ & $.22^{* *}$ & $.29^{* *}$ & $.37^{* *}$ & $.29^{* *}$ \\
\hline Quiz - Politiker & $.52^{* *}$ & $.52^{* *}$ & $.54^{* *}$ & $.47^{* *}$ & $.58^{* *}$ & $.40^{* *}$ \\
\hline Quiz - Issues & $.47^{* *}$ & $.49^{* *}$ & $.38^{* *}$ & $.51^{* *}$ & $.51^{* *}$ & $.49^{* *}$ \\
\hline Politiker - Issues & $.49^{* *}$ & $.50^{* *}$ & $.46^{* *}$ & $.50^{* *}$ & $.59^{* *}$ & $.43^{* *}$ \\
\hline
\end{tabular}

schlägt sich in etwas besserer Kenntnis ihrer Positionen nieder, während die Positionen der anderen Parteien im Westen häufiger zutreffend benannt werden. Allerdings ist das durchschnittliche Kenntnisniveau nur bei wenigen Parteien überzufällig gut, wenn man bedenkt, dass die Ratechance bei 50 Prozent liegt. Bei den Befragten mit türkischem Migrationshintergrund, besonders bei jenen ohne deutsche Staatsbürgerschaft ist das Kenntnisniveau erneut deutlich geringer - tendenzielle Ausnahmen finden sich wiederum bei Themen mit „Betroffenheitsverdacht“, das heißt der EU-Vollmitgliedschaft der Türkei und dem Kommunalwahlrecht für Nicht-EU-Ausländer bei den größeren westlichen Parteien.

Die Frage, inwieweit die Kenntnisse der unterschiedlichen Aspekte des abgefragten Wissens untereinander zusammenhängen, kann die Korrelationstabelle (siehe Tabelle 5) beantworten. Der wichtigste Befund hierzu ist, dass in allen Befragtengruppierungen die Quizfragen zu Strukturen und Regeln mit den Fragen zu Parteizugehörigkeiten der Politiker und Issuepositionen der Parteien relativ stark zusammenhängen, ebenso wie letztere beiden untereinander. Lediglich die einzelne Frage zur Nationalhymne zeigt deutlich geringere Zusammenhänge mit den anderen Skalen. Daher wird dieses Item bei den folgenden Analysen weggelassen, während die anderen alle zu einer gemeinsamen Wissensskala zusammengefasst werden. Kennwerte zu deren Verteilungen sind in Tabelle 6 abgebildet. Von Interesse ist hier besonders, dass die Gesamtskala durchgehend hohe Reliabilitätswerte erreicht, bei den Befragten mit Migrationshintergrund jedoch etwas schlechter misst als bei den Einheimischen.

\begin{tabular}{|l|rrr|rrr|}
\hline Tabelle 6: Gesamtskala Politisches Wissen \\
\hline & \multicolumn{7}{|c|}{ einheimische Deutsche } & \multicolumn{3}{c|}{ türkischer Migrationshintergrund } \\
\cline { 2 - 7 } Range: $0-43$ & gesamt & West & Ost & gesamt & $\begin{array}{c}\text { deutsche } \\
\text { Staats- } \\
\text { angehörigkeit }\end{array}$ & $\begin{array}{c}\text { keine deutsche } \\
\text { Staats- } \\
\text { angehörigkeit }\end{array}$ \\
\hline Mittelwert & 22.77 & 23.06 & 21.55 & 15.71 & 18.52 & 14.35 \\
Standardabweichung & 7.81 & 7.81 & 7.69 & 7.05 & 6.72 & 6.84 \\
Schiefe & -.17 & -.21 & -.04 & .18 & .58 & .08 \\
Modalwert & 17 & 17 & 16 & 20 & 17 & 20 \\
Median & 23 & 23 & 21 & 15 & 17 & 15 \\
Minimum & 1 & 1 & 3 & 1 & 2 & 1 \\
Maximum & 42 & 42 & 39 & 35 & 35 & 35 \\
Reliabilität alpha & .86 & .86 & .86 & .73 & .67 & .75 \\
\hline Quelle: Eigene Berechnung. & \multicolumn{7}{|c}{} \\
\hline
\end{tabular}




\section{Determinanten politischen Wissens}

Als förderlich für den individuellen politischen Kenntnisstand erweisen sich in der bivariaten Analyse gewisse Voraussetzungen an Fähigkeiten (wie Merkfähigkeit) und das Training dieser Kompetenzen in Schule und Ausbildung, motivationale Faktoren (zum Beispiel das Interesse an Politik, die aktive Rezeption politischer Medieninhalte), aber auch Opportunitätsstrukturen (Konfrontation mit politischen Inhalten als Nebenprodukt anderer Aktivitäten, zum Beispiel in Vereinen). Darüber hinaus sind Sozialisation und verfügbare Ressourcen von Bedeutung. Wenig überraschend sind nahezu sämtliche wissensförderlichen Determinanten (mit Ausnahme der Merkfähigkeit) in der einheimischen Bevölkerung etwas stärker ausgeprägt als in der Population mit Migrationshintergrund (siehe Tabelle 7 , Spalten bivariat).

In der multivariaten Analyse zeigen sich sowohl für die Befragten mit deutschem Hintergrund als auch für die mit türkischem Migrationshintergrund erfreulich gute Varianzaufklärungen mit $\mathrm{r}^{2}=.44$ beziehungsweise $\mathrm{r}^{2}=.49$. Dabei verschwindet allerdings die Bedeutung der Mitgliedschaften sowie der Sozialisation. Als besonders erklärungskräftig verbleiben das Interesse an Politik und die Rezeption politischer Medieninhalte sowie die

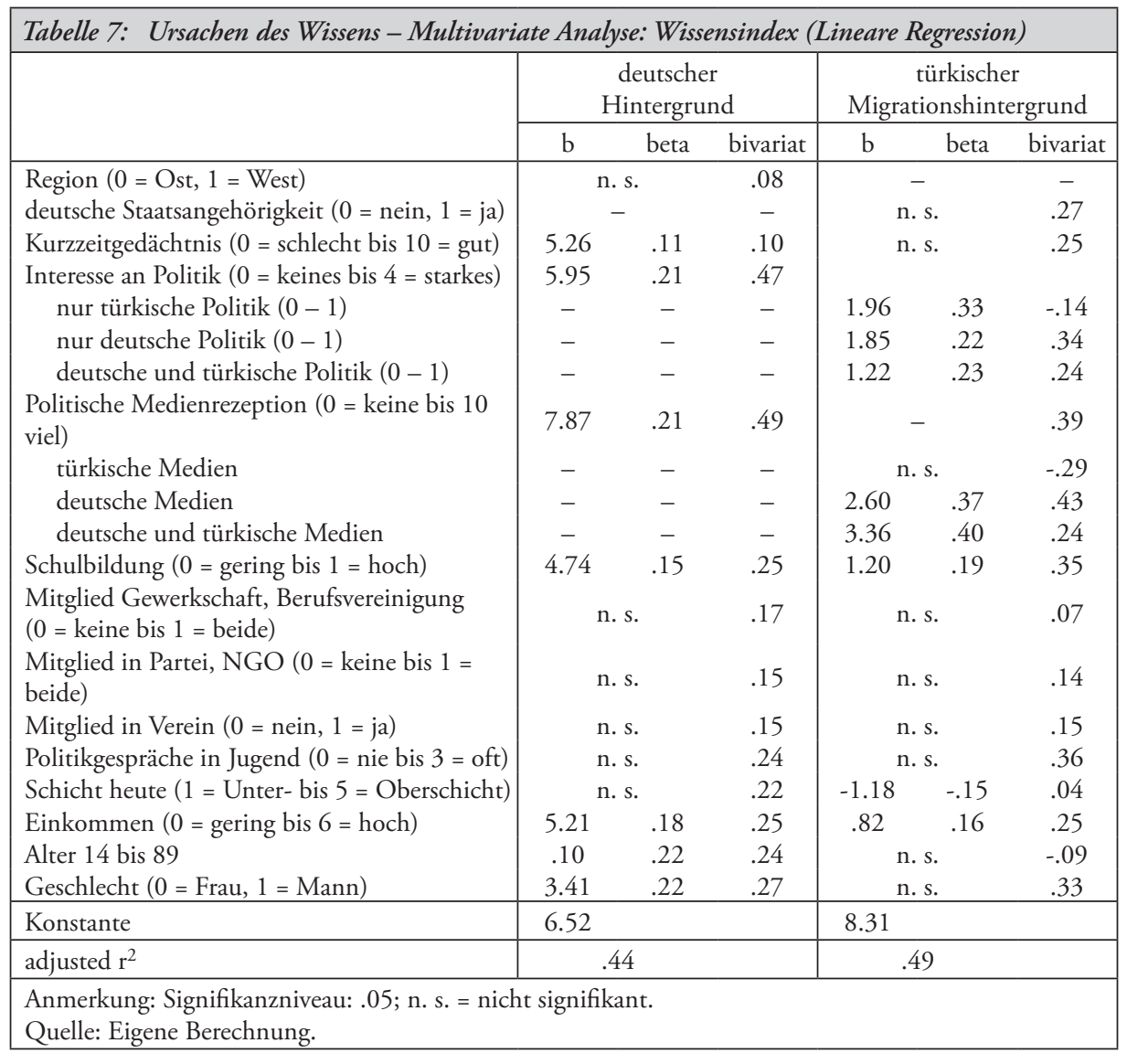


Schulbildung und Einkommen beziehungsweise Schicht. (Infolge der geringen Befragtenzahl sind die multivariaten Befunde zur Migrantenpopulation allerdings nicht sehr robust).

\section{Folgen politischen Wissens}

Zur politischen Legitimität wird in der Theorie angenommen, dass mit steigendem Kenntnisstand auch das Verständnis für demokratische Politik, für politische Probleme sowie Möglichkeiten und Grenzen der Politik wächst. Dies müsste in größerer politischer Unterstützung resultieren. Bei den Befragten mit deutschem Hintergrund ist dies auch deutlich der Fall: Je größer der Wissensstand ist, desto stärker wird die Idee der Demokratie befürwortet (Pearsons r .30), desto größer fällt die Zufriedenheit mit der Umsetzung der Demokratie in Deutschland aus (.18), und desto positiver werden selbst die Leistungen der amtierenden Bundesregierung (.14) beurteilt. Bei den Befragten mit türkischem Migrationshintergrund zeigen sich hier allerdings keine nennenswerten Effekte.

Der theoretisch naheliegende und erwartbare Einfluss auf das politische Effektivitätsbewusstsein ist dagegen in beiden Populationen sehr deutlich: Je mehr eine Person objektiv über Politik weiß, desto besser ist auch die Selbsteinschätzung ihres politischen Verständnisses und ihrer Fähigkeiten, politisch zu agieren (Pearsons r von über .40 in beiden Populationen).

Der Wissensstand korrespondiert schließlich auch mit der politischen Beteiligung, und zwar sowohl bei Formen konventioneller Einflussnahme (Pearsons .28 bei einheimischen und .22 bei Migranten) als auch bei legalem Protest (Pearsons $\mathrm{r} .30$ beziehungsweise .39).

Abschließend soll noch auf einen Einwand eingegangen werden, der in der früheren Forschung wiederholt artikuliert wurde und nicht unwesentlich mit dafür verantwortlich ist, dass es in Deutschland so wenige Erhebungen zu politischem Wissen gibt. Danach sei es überflüssig, politisches Wissen zu ermitteln, weil dieses so stark von Schulbildung und politischem Interesse geprägt wird - beides Variablen, die ebenfalls für Partizipation von zentraler Bedeutung sind. Mithin: politisches Wissen habe neben diesen beiden Merkmalen überhaupt keinen eigenständigen Effekt auf politische Partizipation und sei somit irrelevant. Die empirische Analyse spricht jedoch klar gegen diese Behauptung (siehe Abbildung 2). In den Wissenstest wurden die genannten Variablen integriert und dies noch durch Hinzunahme des Effektivitätsbewusstseins verschärft: Gegenüber dem bivariaten Effekt reduziert sich in der multivariaten Analyse der Wert für das politische Wissen. Aber der verbleibende Effekt von beta $=.17$ beziehungsweise beta $=.16$ ist immerhin etwa gleich groß wie der von politischem Interesse (.15 beziehungsweise .24) und nur etwas schwächer als der Effekt der Selbsteinschätzung (.27 beziehungsweise .18). Politisches Wissen hat also eine durchaus eigenständige Bedeutung für die politische Partizipationsbereitschaft.

\section{Politisches Wissen in Deutschland: ein Feld mit großem Forschungsbedarf}

Die wichtigsten Schlussfolgerungen dieser Exploration in das Feld des politischen Wissens in Deutschland können wie folgt zusammengefasst werden:

(1) Es besteht eine große Spannbreite innerhalb des Wissens zu politischen Fakten von nahezu 90 Prozent bis unter fünf Prozent - je nach Themenbereich, ohne dass sich jedoch bereits klare Kriterien dafür herauskristallisiert hätten. 


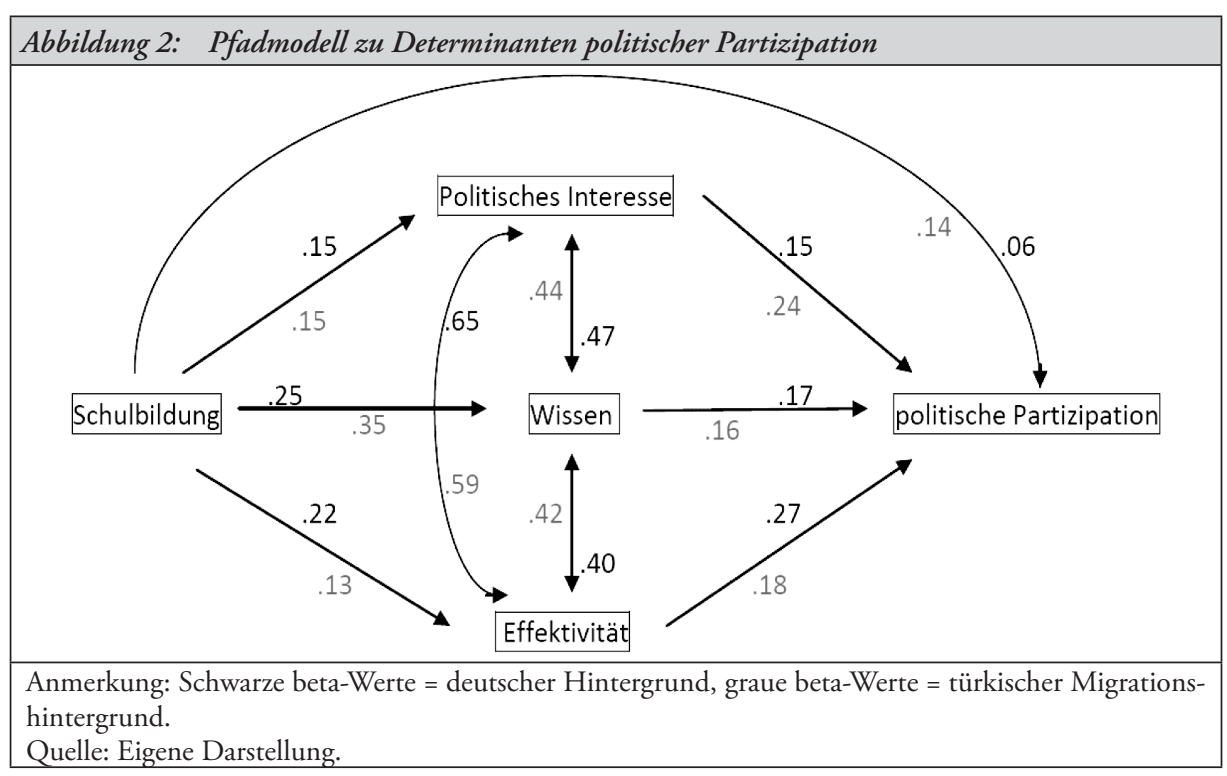

(2) Einheimische Deutsche in West und Ost verfügen im Durchschnitt über ein etwas besseres politisches Faktenwissen als Befragte mit türkischem Migrationshintergrund. Sofern letztere die deutsche Staatsbürgerschaft haben, reduziert sich dieser Unterschied - was hier Ursache, was Wirkung ist, muss zunächst offen bleiben. Dazu sind Longitudinalstudien erforderlich.

(3) Subjektive Betroffenheit und Identifikation sind wichtige Faktoren für den Wissenserwerb. Zudem wird auch die Rolle der Schulbildung und der Medienexposition deutlich.

(4) Die befragten Personen türkischen Hintergrunds weisen überwiegend einen starken Bezug zur Türkei auf und eine geringere Identifikation mit Deutschland. Dementsprechend konzentriert sich ihr politisches Interesse und ihre Mediennutzung auch stärker auf den türkischen Kontext als auf den deutschen, was dem Erwerb von politischem Faktenwissen zu Deutschland abträglich ist. Darüber hinaus sind auch weitere für die politische Bildung relevante Ressourcen in der türkischstämmigen Population geringer verbreitet als in der deutschen, vor allem höhere Schulbildung und besseres Einkommen.

(5) Politisches Wissen erweist sich überwiegend als relevant für politische Urteilsbildung und durchgängig als bedeutsam für politische Partizipation.

Eine Vielzahl von Fragen und Problemen sind mit diesen Befunden noch gar nicht angesprochen. So ließ sich die Frage nach Generalisten versus Spezialisten auf der Grundlage dieser Erhebung leider nicht eindeutig beantworten - dazu werden offenbar Untersuchungen mit Spezialpopulationen und Spezialthemen vonnöten sein, da es im Rahmen einer Studie ohne massive Überforderung der Geduld der Befragten nicht möglich ist, genügend Wissensfragen zu stellen, um diese Problematik zu durchdringen.

Auch ist die Ermittlung von Faktenwissen nur ein erster Schritt. Sie sollte künftig ergänzt werden durch Studien, die weitergehend auf prozedurale Fähigkeiten und das Verständnis von politischen Prozessen sowie Zusammenhängen zielen und in der Lage sind, die Qualität politischer Partizipation (zum Beispiel im Sinn des Erfolgs von Einflussnahme) 
kenntnisreicher und kenntnisarmer Populationen zu vergleichen. Dafür sind auch experimentelle Ansätze nötig. Zur Einordnung des Niveaus des Faktenwissens in Deutschland sind außerdem international vergleichende Studien erforderlich.

Sämtliche Analysen von Befragten mit Migrationshintergrund kranken an der geringen Befragtenzahl; um hier Abhilfe zu schaffen, bedarf es stark überproportionaler Samples, da ansonsten multivariate Analysen mit vernünftiger Fallzahl kaum möglich sind. In Bezug auf Migranten wären zudem Panelerhebungen sinnvoll, um Kausalitätsprozessen zwischen Wissenserwerb, Identifikation mit dem Aufnahmeland und Erwerb der Staatsangehörigkeit des Aufnahmelandes auf die Spur zu kommen.

Insgesamt stellt sich die Ermittlung politischen Wissens in der Bevölkerung, der Ursachen und Folgen als zwar diffiziles, aber auch äußerst interessantes, wichtiges und im Hinblick auf das Erkenntnisinteresse lohnendes Forschungsgebiet dar, das in Deutschland noch einen erheblichen Ausbaubedarf hat.

\title{
Was bewegt Politiker mit Migrationshintergrund? Befunde aus deutschen Großstädten*
}

\author{
Constanze Schmitz und Andreas M. Wüst
}

„Türken, bringt euch mehr ein!“, forderte die CDU-Politikerin Aygül Özkan im April in einem Interview mit Spiegel Online. ${ }^{1}$ Wenige Tage zuvor war die Politikerin mit türkischem Migrationshintergrund eine Stufe auf der Karriereleiter nach oben geklettert. Christian Wulff, damals noch Ministerpräsident in Niedersachsen, bot der Abgeordneten der Hamburger Bürgerschaft den Posten der Sozialministerin in seinem Kabinett an. Damit wurde Özkan die erste türkischstämmige Ministerin Deutschlands - aus den Reihen der CDU. Das erregte Aufsehen, und obwohl sie Sozialministerin werden sollte, behandelten die vielen Fragen von Journalisten, denen sich Frau Özkan stellte, nicht das breite Spektrum des Politikfelds Soziales, sondern es ging fast ausschließlich um ihre Person und vermeintliche Gruppenzugehörigkeit. Wie kommt eine Muslimin dazu, in einer christlichen Partei politisch aktiv zu sein? Müsste sie nicht eigentlich bei der SPD oder bei den Grünen sein? Welche Integrationspolitik gedenke sie zu machen, und was hielt sie vom Integrationsstatus ihrer „Landsleute"?2

* Die Forschungsanstrengungen wurden durch die Volkswagen-Stiftung ermöglicht. Dies wird von den Autoren dankbar angezeigt.

1 Vgl. das Interview Aygül Özkans mit Anna Reimann, Neue Ministerin Özkan - „Türken, bringt euch mehr ein“, in: Spiegel Online vom 23. April 2010, http://www.spiegel.de/politik/deutschland/0,1518,690702,00.html (Abruf am 28. November 2011).

2 Vgl. ähnliche Inhalte bei Ulrich Exner, Hilfe, diese Muslima ist gar keine Christin!, in: Welt Online vom 26. April 2010, http://www.welt.de/politik/deutschland/article7346946/Hilfe-dieseMuslima-ist-gar-keine-Christin.html; „Es lohnt sich, sich anzustrengen“, in: Berliner Morgenpost vom 27. April 2010, http://www.morgenpost.de/printarchiv/seite3/article1296879/Es-lohntsich-anzustrengen.html; Stephanie Jungholt, Frau Özkan, warum sind Sie als Muslimin in der 